\title{
Speaking in Two Voices: Mehrsprachige Gedichte im Fremdsprachenunterricht
}

\author{
Mag. ${ }^{a}$ Dr. ${ }^{\text {in }}$ Maria Magdalena Mayr-Hueber \\ Institut für Fachdidaktik, Bereich Didaktik der Sprachen \\ Universität Innsbruck
}

\section{Einleitung}

Der vorliegende Artikel basiert auf einer von der Autorin im Rahmen ihrer Dissertation (Mayr 2014) durchgeführten empirischen Studie über die Verwendung zweisprachiger Gedichte im mehrsprachig ausgerichteten Fremdsprachenunterricht und präsentiert, ausgehend von einem Überblick über die Zielsetzungen und den Ablauf der Untersuchung, ausgewählte Ergebnisse, welche im Bereich der Sprach- und Literaturdidaktik von Relevanz sein können. Die Studie untersuchte die Reaktionen von SchülerInnen auf die Bearbeitung englisch-spanischer Gedichte in einem mehrsprachig ausgerichteten Fremdsprachenunterricht und wurde im Zeitraum von November 2012 bis Juni 2013 durchgeführt.

\section{Theoretische Grundlagen}

Die Studie situiert sich durch ihr Forschungsinteresse am Schnittpunkt verschiedener Wissenschaftsdisziplinen und kann somit als interdisziplinär bezeichnet werden. Das folgende Kapitel soll kurz die Grundpfeiler dieser unterschiedlichen theoretischen Hintergründe umreißen. Es handelt sich dabei einerseits um verschiedene Aspekte einer mehrsprachig ausgerichteten Didaktik, andererseits um die Auseinandersetzung mit Literatur im Fremdsprachenunterricht. 


\subsection{Ansätze einer mehrsprachigen Fremdsprachendidaktik}

In der Einleitung zu Beyond Bilingualism: Multilingualism and Multilingual Education beschreiben Cenoz \& Genesee (1998) mehrsprachige Gemeinschaften und Personen als die weltweit typische Norm. Auch in der schulischen Realität wird die Notwendigkeit von „(mehr)sprachlichem Wissen und Sprachenbewusstheit“ aufgrund der lebensweltlichen Mehrsprachigkeit der SchülerInnen immer größer (Blell 2012: 11). Gerade im schulischen Kontext lassen sich zahlreiche Konzepte identifizieren, welche auf teilweise sehr unterschiedliche Art darauf abzielen, verschiedene Formen von Mehrsprachigkeit in die Schulrealität zu integrieren. Im anglophonen Bereich beispielsweise, insbesondere in den USA, bezieht sich der Begriff multilingual education häufig auf die aktive Einbindung von Herkunftssprachen in den Unterricht, auf die Förderung der individuellen sprachlichen und kulturellen Identität der Kinder und Jugendlichen sowie auf die erfolgreiche persönliche Einbindung von neu immigrierten SchülerInnen in die Gemeinschaft (vgl. u.a. García et al. 2006; López-Gopar et al. 2013; Schecter \& Cummins 2003; van der Walt 2013). Häufig wird dabei auf die Notwendigkeit gepocht, die verschiedenen Muttersprachen der SchülerInnen auf konstruktive Weise in den (Englisch-)Unterricht einzubauen und dadurch das Selbstwertgefühl und damit auch die Bildungszugänglichkeit der jungen Menschen zu stärken. Darüber hinaus wird in vielen Fällen auf einen kulturellen und persönlichen Lernprozess abgezielt, der durch kreative Schreib- oder Lernprozesse gefördert werden soll (vgl. u.a. Cahnmann 2006; Cummins 2006; López-Gopar et al. 2013).

Ein etwas anderes Verständnis von mehrsprachigem Lernen hat sich in den letzten Jahren und Jahrzehnten an Forschungsstätten v.a. in Deutschland aber auch anderen europäischen Ländern herausgebildet. Die Entwicklung einer Mehrsprachigkeitsdidaktik gründet sich hier stärker auf die im schulischen Kontext vorgelernten Sprachen, welche den Lernenden zusätzlich zur Muttersprache oder zu den Muttersprachen noch zur Verfügung stehen, wie diese verschiedenen Sprachkonstellationen zusammenwirken, um eine übergreifende Sprachkompetenz zu ergeben, und wie die dadurch zur Verfügung stehenden Sprachen sich positiv auf das Erlernen einer weiteren Sprache auswirken können (vgl. zahlreiche Beiträge von Meißner 1995 bis Grünewald \& Sass 2014: 27). Die sprachliche

Barbara Hinger (Hg.), Zweite „Tagung der Fachdidaktik“ 2015.

Sprachsensibler Sach-Fach-Unterricht - Sprachen im Sprachunterricht.

(C) 2016 innsbruck university press, ISBN 978-3-903122-51-2, DOI 10.15203/3122-51-2 
Komponente wird hier - unter anderem durch stark formalisierte und geregelte Modelle - stärker in den Vordergrund gerückt, obwohl auch inter- und soziokulturelle Elemente in die Überlegungen zur Mehrsprachigkeit mit einfließen. Es ist wohl unter anderem auf die Vielfalt und die ambitionierten Zielsetzungen von mehrsprachiger Bildung zurückzuführen, wenn Cenoz \& Genesee (1998: viii) bemerken, „multilingual education is not an easy enterprise“. Dennoch ist mehrsprachige Erziehung und Bildung, in welcher Form auch immer, ein äußerst lohnendes Unterfangen, da Mehrsprachigkeit nicht nur in Europa, sondern auch in anderen Ländern der Welt Positives mit sich bringen kann und Diversität heutzutage stärker als Vorteil wahrgenommen wird denn als Problem (Cenoz \& Gorter 2010: 50).

Auf welche Weise versucht nun die hier beschriebene Studie, Mehrsprachigkeit in die Schulrealität einzubinden? Vorrangiges Ziel war es, eine schulische Situation zu untersuchen, in der mehrere in der Lebenswelt der SchülerInnen verankerte Sprachen gleichzeitig, übergreifend und kreativ verwendet werden. Sowohl die lebensweltlichen Sprachen als auch die Schulfremdsprachen der TeilnehmerInnen wurden also im Unterricht aktiviert und miteinander in Verbindung gesetzt. Somit baut die Studie gleichzeitig auf verschiedenen Konzepten der Mehrsprachigkeit auf und versucht, diese auf schlüssige Weise und am konkreten Beispiel literarischer Texte miteinander zu verknüpfen.

\subsection{Literarische Texte im Fremdsprachenunterricht}

Die Textgrundlagen der hier beschriebenen Studie bilden ohne Zweifel authentische literarische Texte, die in einer Unterrichtssituation bearbeitet wurden. Dennoch liegt das Erkenntnisinteresse der Untersuchung nicht überwiegend auf der Art der Bearbeitung der Texte oder auf dem didaktischen Gehalt der Texte selbst; vielmehr fokussieren die Forschungsfragen, wie die SchülerInnen persönlich und individuell mit den Texten umgehen. Daher sollen hier vorrangig jene Überlegungen dargelegt werden, welche zur Verwendung von literarischen Texten in der hier beschriebenen Studie führten. Darüber hinaus wird kurz jene literaturdidaktische Ausrichtung vorgestellt, die den verwendeten Unterrichtsentwürfen zugrunde liegt.

Barbara Hinger (Hg.), Zweite „Tagung der Fachdidaktik“ 2015.

Sprachsensibler Sach-Fach-Unterricht - Sprachen im Sprachunterricht.

(C) 2016 innsbruck university press, ISBN 978-3-903122-51-2, DOI 10.15203/3122-51-2 
Da die SchülerInnen unter anderem dazu angeregt werden sollten, eigene Erfahrungen auf Basis der bearbeiteten Texte zu reflektieren, liefern Konstrukte von Sexl (2003) hier wichtige Gründe: Er beschreibt literarische Texte als wichtiges Werkzeug zur Schulung des Interpretierens und des Verstehens von Situationen im realen Leben (Sexl 2003: 266). Laut Sexl (2003: 267) können eigene Handlungsweisen durch ihre Spiegelung in einem literarischen Text besser wahrgenommen und das Spektrum möglicher Verhaltensweisen kann erweitert werden. Auch ethische Überlegungen zu verschiedenen Handlungen können dadurch tragend werden (2003: 268). Darüber hinaus argumentiert Sexl (2003: 267f.), dass literarische Texte sich besser als andere Texte für die Bewusstmachung von Sprachverwendung eignen und sprachliche Alternativen liefern, was für die hier beschriebene Arbeit von hoher Bedeutung ist, da sie sich explizit mit der Sprachverwendung der AutorInnen, aber auch der Lernenden selbst auseinandersetzt. In seiner Arbeit „Literatur und Erfahrung“ argumentiert Sexl die Notwendigkeit einer solchen Schulung der Interpretations- und Verstehensfähigkeit in Bezug auf den konkreten Kontext des Gesundheitswesens, z.B. in der Interaktion zwischen ärztlichem Personal und Pflegepersonal oder in der Interpretation von Krankheitsbildern (Sexl 2003: 267f.), welche sich nicht immer offensichtlich erkennen oder erschließen lassen, sondern einer umfassenden Beleuchtung aus verschiedenen Blickwinkeln bedürfen, um als solche erkannt zu werden. Auch in der Verhandlung von sprachlichen und vor allem kulturellen Werten bzw. Identitäten sowie in der interkulturellen Verständigung kommt der Interpretation und dem Verstehen von verschiedenen Sichtweisen hohe Bedeutung zu. Demzufolge spielen solche Interpretations- und Verstehensprozesse auch im Kontext der hier beschriebenen Studie eine große Rolle, und zwar in Bezug auf die literarischen Textgrundlagen selbst sowie auch auf die Verhandlungsprozesse zwischen den SchülerInnen im schulischen Kontext. Darüber hinaus ist die Interpretation von eigenen Gefühlen oder Einstellungen gegenüber Sprachen und kulturellen Einflüssen bzw. denen anderer Personen gerade in Bezug auf die behandelten Texte besonders wichtig.

Über diese grundlegenden Überlegungen zur Verwendung von literarischen Texten in der hier beschriebenen Studie hinaus wurde auch in der Konzeption der Studie auf Prinzipien der Literaturdidaktik zurückgegriffen. Eine wichtige

Barbara Hinger (Hg.), Zweite „Tagung der Fachdidaktik“ 2015.

Sprachsensibler Sach-Fach-Unterricht - Sprachen im Sprachunterricht.

(C) 2016 innsbruck university press, ISBN 978-3-903122-51-2, DOI 10.15203/3122-51-2 
Rolle kam hier der Rezeptionsästhetischen Literaturdidaktik (vgl. Bredella \& Burwitz-Melzer 2004) zu, welche einen persönlichen Zugang zum literarischen Text postuliert - und zwar auf kognitiver sowie emotionaler Ebene - und sowohl den/die LeserIn als auch den Text selbst im Lese- und Interpretationsprozess anerkannt sehen möchte. Beide Faktoren sind für diese literaturdidaktische Ausrichtung in der Bedeutungsentwicklung bestimmend und nehmen eine zentrale Rolle ein. Damit soll nicht nur die Empathiefähigkeit des Lesepublikums, sondern auch dessen Urteilsvermögen (Bredella \& Burwitz-Melzer: XV) geschult werden, was - wie bereits erwähnt - für den Kontext der hier beschriebenen Studie wesentlich ist, wie weiter unten ausgeführt wird. Demzufolge wurde die Rezeptionsästhetische Literaturdidaktik als Basis für die Unterrichtsentwürfe, welche im Rahmen der Studie umgesetzt wurden, ausgewählt. Beispiele für den rezeptionsästhetischen Fokus finden sich durchgehend in allen drei Unterrichtsplanungen; unter anderem lässt sich hier auf die offene Erstrezeption (A2.1) ${ }^{1}$, den häufigen Meinungsaustausch zum Inhalt der Texte (u.a. A1.6, A2.3, A2.7, A3.2) sowie auf das selbständige Verfassen von kreativen Texten bzw. einer Collage in Reaktion auf die bearbeiteten Gedichte (A1.7, A2.8, A3.6) verweisen.

\section{Forschungsdesign}

Die Studie sucht Einblick in einen Bereich zu gewähren, der noch weitgehend unerforscht ist, und verortet sich daher im qualitativen Forschungsparadigma, denn für explorative Vorgehensweisen, wie sie im Fall der hier beschriebenen Studie genutzt werden, eignen sich qualitative Forschungsmethoden laut Dörnyei (2007: 39ff.) ganz besonders. Ziel der Studie war also nicht die Verifizierung oder Falsifizierung bereits bestehender Hypothesen, sondern vielmehr eine tiefgehende Erforschung des Wie und Warum von Denkprozessen der TeilnehmerInnen. Im Zentrum der Studie standen folgende Forschungsfragen:

1 Die Kennzeichnungen $A 2.1$ etc. beziehen sich auf die einzelnen Unterrichtsschritte in den Stundenbildern (s. Kap. 5.1, 5.2, 5.3). 
- Welche Denk- und Verstehensprozesse werden bei der Bearbeitung von mehrsprachigen literarischen Texten in den SchülerInnen ausgelöst?

- (Inwiefern) Unterscheiden sich diese Prozesse bei Lernenden mit lebensweltlich mehrsprachigem und mehrkulturellem Hintergrund im Vergleich zu monolingual/ monokulturell sozialisierten Lernenden?

Die erste der beiden Forschungsfragen ist bewusst allgemein gehalten. Die zweite Frage widmet sich der Verbindung zwischen dem lebensweltlichen Sprachund Kulturhintergrund der TeilnehmerInnen und deren Rezeption der Texte. Sie versucht also, mögliche Unterschiede in der Herangehensweise an einen Text zu zeigen und betrachtet die TeilnehmerInnen unter dem Blickwinkel ihrer individuellen sprachlichen und kulturellen Hintergründe.

\subsection{Forschungsmethodik, Analyseinstrumente und Aufbau der Studie}

Wie aus den Forschungsfragen unschwer zu erkennen ist, wird in der Studie vorrangig die Verbindung der aus den Unterrichtseinheiten gewonnenen Einblicke mit dem sozialen und lebensweltlichen Hintergrund der SchülerInnen untersucht. Um eine Basis für die Beantwortung der Forschungsfragen zu schaffen, wurde in einer Reihe von drei Unterrichtseinheiten im Spanischunterricht zweier Lerngruppen der Sekundarstufe II mit englisch-spanischen bilingualen Gedichten und eigens für die Studie entwickelten Unterrichtsmaterialien gearbeitet. Im Rahmen der Untersuchung konnten Einsichten in die Vielschichtigkeit der Denkprozesse und Erfahrungen der SchülerInnen mit den Texten erhoben werden.

Ein weiterer methodologischer Aspekt der hier beschriebenen Studie ist die Aktionsforschung, welche zwar als umstritten gilt, für die Untersuchung jedoch von hoher Bedeutung ist, da die Forscherin auch gleichzeitig selbst als Lehrperson tätig war und die TeilnehmerInnen in den zu analysierenden Unterrichtseinheiten unterrichtete. Der einzigartige Synergieeffekt aus Theorie und Praxis, der sich daraus ergibt, kommt dem Forschungsprojekt insofern zugute, als die Forscherin auch gleichzeitig Expertin auf ihrem Gebiet ist und das Umfeld, aber auch die Absichten der Studie kennt. In einem weiteren Kontext lässt sich die Aktionsforschung im Bereich des Design-Based Research einordnen, welcher vor-

Barbara Hinger (Hg.), Zweite „Tagung der Fachdidaktik“ 2015.

Sprachsensibler Sach-Fach-Unterricht - Sprachen im Sprachunterricht.

(C) 2016 innsbruck university press, ISBN 978-3-903122-51-2, DOI 10.15203/3122-51-2 
rangig Lehr- und Lernumgebungen erforscht und zu verbessern sucht (Reinmann 2015). In einem solchen Untersuchungsdesign sollen nicht nur neue Lernkontext und deren Implementationsmöglichkeiten verbessert werden, sondern die Untersuchung soll auch zu einem besseren theoretischen Verständnis über Lehren und Lernen führen. Dafür wird häufig ein Methoden-Mix verwendet (vgl. Reinmann 2015), was auch in der hier beschriebenen Studie, vor allem im Bereich der Datenerhebung, der Fall ist.

Zur Datenerhebung wurden verschiedene Forschungsinstrumente verwendet, um einen möglichst umfassenden Blick auf das Unterrichtsgeschehen und die Reflexionseinsichten der TeilnehmerInnen werfen und sie in ihrer Komplexität erfassen zu können. Einerseits wurden die Unterrichtseinheiten videographiert, um Schlüsselstellen im Unterrichtsgeschehen identifizieren und zeitliche Abfolgen nachvollziehen zu können sowie um eine spätere Kommentierung des Geschehens durch die TeilnehmerInnen zu ermöglichen. Andererseits wurden zwei von den SchülerInnen während des Unterrichtsgeschehens selbst verfasste Texte zu ihrer eigenen sprachlichen und kulturellen Situation sowie aus der Perspektive einer der Figuren (s. Ablaufbeschreibung der Unterrichtseinheiten) analysiert. Darüber hinaus wurde nach den ersten beiden Unterrichtseinheiten jeweils eine Einheit des sogenannten Stimulated recall Verfahrens (vgl. u.a. Dörnyei 2007; Gass \& Mackey 2000) durchgeführt, welches die Denkprozesse der einzelnen TeilnehmerInnen während der Ausführung verschiedener Aktivitäten dokumentieren sollte. Bei der introspektiven Forschungsmethode des Stimulated recall handelt es sich um ein Laut-Denk-Protokoll, das jedoch nicht während der eigentlichen Aktivität, sondern anhand eines mit der fokussierten Aktivität verbundenen Stimulus (z.B. Videosequenz, Audiodatei, schriftliche Dokumente aus der Unterrichtssituation etc.) (Dörnyei 2007: 148f.) im Nachhinein erhoben wird. Als Stimuli wurden in der hier vorgestellten Studie einerseits von den SchülerInnen selbst verfasste Texte und andererseits ausgewählte Videosequenzen aus den Unterrichtseinheiten verwendet. Nach Durchführung der Unterrichtsreihe fanden halbstrukturierte Einzelinterviews mit den TeilnehmerInnen statt, in denen diesen die Möglichkeit geboten wurde, über ihre Erfahrungen während der Unterrichtseinheiten zu sprechen und diese zu reflektieren. Somit ergibt sich folgender Untersuchungsablauf:

Barbara Hinger (Hg.), Zweite „Tagung der Fachdidaktik“ 2015.

Sprachsensibler Sach-Fach-Unterricht - Sprachen im Sprachunterricht.

(C) 2016 innsbruck university press, ISBN 978-3-903122-51-2, DOI 10.15203/3122-51-2 


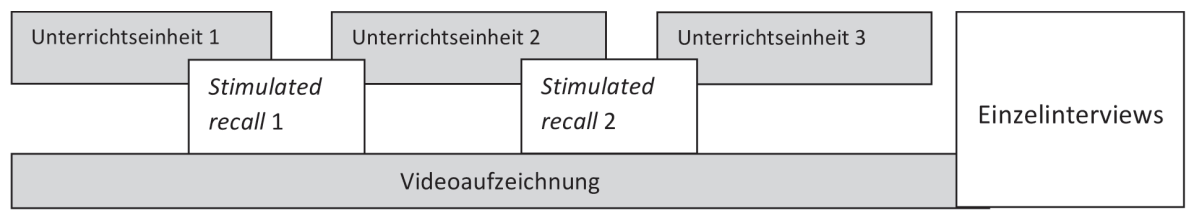

Abb. 1: Aufbau der Studie

Die Datensätze aus den vier eben besprochenen Erhebungsinstrumenten wurden mithilfe der qualitativen Inhaltsanalyse nach Mayring (2010) und unter Heranziehung des Analyseinstruments MAXQDA analysiert und anschließend auf der Basis sich überschneidender Ergebniskategorien zwischen den einzelnen Datensätzen trianguliert. Da für die hier beschriebene Studie noch keine theoretischen Konzepte vorliegen, auf die zurückgegriffen hätte werden können, wurden induktiv Kategorien erarbeitet, wie in wenig untersuchten Forschungsbereichen üblich (vgl. u.a. Mayring 2010). Auf diese Weise konnte ein möglichst breites Spektrum an Reaktionen berücksichtigt und eine umfassende Beantwortung der Forschungsfragen ermöglicht werden. Die Triangulation fand auf Basis sich überschneidender Ergebniskategorien statt und sollte nicht der wechselseitigen Überprüfung von Resultaten dienen, sondern eine Erkenntniserweiterung mit sich bringen (Flick 2008: 27). Die induktiven Kategorien, welche sich aus den verschiedenen Datensätzen ergaben, beziehen sich beispielsweise auf die Wahrnehmung der Zweisprachigkeit in den Gedichten, den mehrsprachigen Schreibprozessen, die Sprachverwendung in den selbst verfassten Texten, die Auseinandersetzung mit den Charakteren oder die Verbindung der Textinhalte mit der eigenen Erfahrungswelt. Insgesamt ergaben sich durch die Triangulation zwölf verschiedene Ergebniskategorien (s. Kap. 6.1).

\subsection{ProbandInnen}

Bei den Lerngruppen handelte es sich einerseits um (ausnahmslos weibliche) Schülerinnen eines österreichischen Realgymnasiums, die Spanisch als Wahlpflichtgegenstand gewählt hatten (11. Schulstufe, Beginn 2. Lernjahr, 18 Schülerinnen), und andererseits um SchülerInnen einer österreichischen Handelsakade- 
mie, die sich für den jahrgangsübergreifenden Freigegenstand Spanisch gemeldet hatten (10. bzw. 11. Schulstufe, Ende 1. Lernjahr, 12 SchülerInnen). Von den insgesamt 30 SchülerInnen, die sich zum Zeitpunkt der Studie im Alter zwischen 15 und 17 Jahren befanden, waren 21 einsprachig mit der Muttersprache Deutsch aufgewachsen; die restlichen neun wiesen einen lebensweltlich mehrsprachigen Hintergrund auf, womit - über das Deutsche hinaus - die Sprachen Bosnisch (2), Finnisch (1), Französisch (1), Italienisch (1), Kroatisch (2), Spanisch (1) und Türkisch (1) als Muttersprachen in der Gesamtgruppe vertreten waren. Das sonstige sprachliche Vorwissen der SchülerInnen in Bezug auf den schulischen Unterricht umfasste die Sprachen Englisch als erste sowie entweder Französisch oder Italienisch als zweite lebende Fremdsprache. Für die Datenerhebung und die Auswertung der Daten konnte aus verschiedenen, mit den Bedingungen des Schulbetriebs zusammenhängenden Gründen jedoch nur eine geringere Anzahl an TeilnehmerInnen herangezogen werden. Nach eingehender Auseinandersetzung mit den Gegebenheiten und Möglichkeiten wurde die Zahl 15 als angemessene TeilnehmerInnenzahl für die Auswertung festgesetzt. Von diesen 15 TeilnehmerInnen wiesen sechs einen lebensweltlich mehrsprachigen Hintergrund auf (Bosnisch (1), Finnisch (1), Italienisch (1), Kroatisch (2), Türkisch(1)); die restlichen neun SchülerInnen waren einsprachig sozialisiert worden.

Um einen Einblick in das Unterrichtsgeschehen zu gewähren, werden im Folgenden die verwendeten Texte sowie der Ablauf der Unterrichtseinheiten und die durchgeführten Aktivitäten dargestellt. Auf dieser Basis können auch die Ergebnisse der konkreten Untersuchungsschritte klar nachvollzogen werden.

\section{Literarische Textgrundlagen}

Als Grundlage der Unterrichtseinheiten wurden vier verschiedene Texte gewählt, die überwiegend aus dem Bereich der Chicano/a-Literatur stammen. ${ }^{2}$ Nach Bruce-Novoa (1982: 12) bezeichnet der Begriff Chicanos bzw. Chicanas Menschen

2 Die Wurzeln der Autorin Rhina P. Espaillat liegen in der Dominikanischen Republik, daher kann sie strenggenommen nicht als Chicana bezeichnet werden, sondern muss mit dem allgemeineren Terminus Latina gefasst werden..

Barbara Hinger (Hg.), Zweite „Tagung der Fachdidaktik“ 2015.

Sprachsensibler Sach-Fach-Unterricht - Sprachen im Sprachunterricht.

(C) 2016 innsbruck university press, ISBN 978-3-903122-51-2, DOI 10.15203/3122-51-2 
mexikanischer Herkunft, die permanent in den USA leben. Bedingt durch ihren geographischen Standort zwischen den USA und Mexiko und durch ihre Geschichte der Vermischung verschiedener ethnischer Gruppierungen und Kulturen kann sich der Großteil der Angehörigen der Chicano/a-Kultur in beiden Sprachen - Englisch und Spanisch - verständigen und sich auch in beiden Kulturen zurechtfinden (Bruce-Novoa 1982: 308). Nach Ette (2005; 2010) können Texte wie die hier verwendeten Gedichte als „Literatur ohne festen Wohnsitz“ (2005: 13) bezeichnet werden, welche sich sowohl auf transkultureller als auch auf translingualer Ebene bewegen und heute längst nicht mehr die Ausnahme eines Literaturkanons darstellen; vielmehr bringen diese Texte eine tiefgreifende Veränderung der (trans)nationalen Literatursysteme mit sich (Ette 2010: 117). Nach Ette (2010: 125) zeichnen sich diese Literaturen durch das Schaffen eines Bewegungs-Raumes aus, indem sie kontinuierlich zwischen verschiedenen kulturellen Gruppierungen, Gesellschaften, Orten und Sprachen oszillieren (vgl. Ette 2005: 14).

Die in der hier beschriebenen Studie verwendeten Texte spiegeln allesamt auf die eine oder andere Art eben diesen Bewegungs-Raum wider - und zwar sowohl auf inhaltlicher als auch auf sprachlicher Ebene. Im Folgenden werden die Texte einzeln kurz dargestellt. Sie alle bieten, wie es Sexl (2003: 269) fordert und wie es sich in den nachfolgenden Absätzen zeigen wird, genügend Interpretationsspielraum, um eigene Erfahrungen adäquat einflechten zu können.

\subsection{She ${ }^{3}$ (Elizondo 1977)}

Der erste bearbeitete Text, verfasst vom Chicano-Schriftsteller Sergio Elizondo, beschreibt den Tagesablauf einer Frau, die verschiedene alltägliche Tätigkeiten entweder mit der englischen oder der spanischen Sprache verbindet. Dabei zeigt sich, dass die Frau gefühlsbetonte und persönlichkeitsbezogene Aktivitäten (z.B. Fühlen, Lieben, Denken) mit dem Spanischen verknüpft, während berufliche und mit dem öffentlichen Raum verbundene Tätigkeiten (Sitzen, Lesen, Autofahren) mit dem Englischen in Verbindung gebracht werden. Der Autor verwendet für

3 s. Anhang 1

Barbara Hinger (Hg.), Zweite „Tagung der Fachdidaktik“ 2015.

Sprachsensibler Sach-Fach-Unterricht - Sprachen im Sprachunterricht.

(C) 2016 innsbruck university press, ISBN 978-3-903122-51-2, DOI 10.15203/3122-51-2 
die Beschreibung der Aktivitäten auch jeweils die Sprache, mit der die Protagonistin diese Tätigkeiten assoziiert. Ihr Leben zwischen zwei Sprachen und Kulturen kommt somit sowohl auf inhaltlicher als auch auf sprachlicher Ebene zum Ausdruck; vermuten lässt sich ein Migrationshintergrund der Protagonistin mit spanischsprachigen Wurzeln und gegenwärtigem Lebensmittelpunkt in den USA.

Das didaktische Potenzial dieses Texts liegt ebenso im inhaltlichen wie im sprachlichen Bereich. Aufgrund der sehr einfachen sprachlichen Strukturen eignet sich dieser Text besonders als Einstieg in die zweisprachige Literaturarbeit sowie zum Verfassen eines ähnlich strukturierten Textes aus individueller Perspektive von SchülerInnen. Eine möglichst hohe Offenheit in der Interpretation, welche Sexl (2003: 269) als Voraussetzung für eine erfolgreiche Verbindung eines Textes mit der eigenen Lebensrealität sieht, ist in diesem Gedicht ebenso gegeben. Mit Zeilen wie „she sits in English“ oder „quiere en español“ regt der Autor das Lesepublikum unwillkürlich zu Überlegungen darüber an, was es denn - sowohl für den Autor als auch für sich selbst - konkret bedeuten könnte, auf Englisch zu sitzen oder auf Spanisch zu lieben.

\subsection{Bilingual/Bilingüe ${ }^{4}$ (Espaillat 1998)}

In Rhina P. Espaillats Text Bilingual/Bilingüe wird der Konflikt zwischen einer Tochter und ihrem Vater dargestellt, in dem es vorrangig um die Sprachverwendung dieser beiden Figuren geht. Hintergrund ist hier vermutlich ebenfalls eine Migrationssituation zwischen einem spanisch- und einem englischsprachigen Lebensraum. Die Tochter möchte ihre beiden Sprachen - Englisch und Spanisch - gleichermaßen verwenden, während der Vater diese streng trennt und auf ein ausschließliches Verwenden der spanischen Sprache im familiären Kontext zu Hause besteht. Sprachlich ist das Gedicht vorwiegend in Englisch gehalten, wobei einige Textpassagen - sozusagen als Übersetzung der entsprechenden englischen Teile - zusätzlich auf Spanisch eingefügt sind. Die Tatsache, dass hier auch ein Generationenkonflikt behandelt wird, lässt den Text als besonders zugänglich für Jugendliche erscheinen, was sich auch in der Analyse der Daten widerspiegelt:

4 s. Anhang 2

Barbara Hinger (Hg.), Zweite „Tagung der Fachdidaktik“ 2015.

Sprachsensibler Sach-Fach-Unterricht - Sprachen im Sprachunterricht.

(C) 2016 innsbruck university press, ISBN 978-3-903122-51-2, DOI 10.15203/3122-51-2 
Das Gedicht wurde von mehreren TeilnehmerInnen aufgrund seiner Realitätsnähe und der Identifikationsmöglichkeit mit der Situation als interessantester Text eingestuft.

\subsection{A Una Niña de Este País ${ }^{5}$ (Espinosa 1953)}

Das dritte Gedicht ist ein traditioneller Folklore-Text, der von Aurelio Macedonio Espinosa 1953 aufgezeichnet wurde. Er erzählt von der Begegnung zwischen einem Mann und einer jungen Frau und ist zum Großteil in dialogischer Form verfasst, wobei der männliche Protagonist hauptsächlich in spanischer Sprache kommuniziert, während die Frau in gebrochenem Englisch antwortet. Der Mann versucht, die Frau für sich zu gewinnen und ihr eine Liebesbezeugung abzuringen, doch die Frau weist ihn auf sehr direkte Art zurück: „Me no like Mexican men." Aus ihrer Sprachverwendung wird klar, dass sie keine englische Muttersprachlerin ist und vermutlich, ebenso wie der Mann, als Immigrantin in einem englischsprachigen Land lebt; im Gegensatz zu ihm weist sie jedoch ihre Wurzeln zurück und bevorzugt die Verwendung des Englischen. Der dialogische Charakter des Textes, welcher dem Gedicht Witz und Spritzigkeit verleiht, sowie die dargestellte Situation - ein versuchter Flirt - erweckten hier das Interesse der TeilnehmerInnen. Der Konflikt zwischen der sprachlichen und kulturellen Anpassung an ein Zielland und der Aufrechterhaltung der Herkunftssprache und/ oder -kultur regte hier die Fantasie und Diskussionsbereitschaft der SchülerInnen an.

\subsection{Un Beso Is Not a Kiss ${ }^{6}$ (Alarcón 1989)}

Das letzte verwendete Gedicht ist der abstrakteste der vier Texte. In einem konstanten Wechsel zwischen Englisch und Spanisch beschreibt der Autor darin den für ihn bedeutenden Unterschied zwischen einem beso und einem kiss. Die Tatsache, dass die beiden Wörter die gleiche Grundbedeutung haben, nämlich

5 s. Anhang 3

6 s. Anhang 4

Barbara Hinger (Hg.), Zweite „Tagung der Fachdidaktik“ 2015.

Sprachsensibler Sach-Fach-Unterricht - Sprachen im Sprachunterricht.

(C) 2016 innsbruck university press, ISBN 978-3-903122-51-2, DOI 10.15203/3122-51-2 
Kuss, tut der Argumentationsführung keinen Abbruch; vielmehr stellt er einen beso als intensiver und sogar gefährlicher dar als einen kiss. Hier steht vor allem ein sprachlicher Aspekt im Vordergrund: die unterschiedliche Besetzung von Wörtern in verschiedenen Sprachen. Das Ziel bei der Verwendung dieses Textes lag hier vor allem in der Erkenntnis, dass Wörter in verschiedenen Sprachen unterschiedliche Konnotationen haben und von Sprechern auch als unterschiedlich wahrgenommen werden können. Der Text selbst fällt jedoch auf gewisse Weise aus der Reihe und stieß in der Studie nur auf mäßige Begeisterung von Seiten der SchülerInnen.

\section{Beschreibung der Unterrichtseinheiten}

Die Studie wurde in drei Unterrichtseinheiten zu je 100 Minuten in zwei verschiedenen Lerngruppen der Sekundarstufe II durchgeführt (zu den ProbandInnen s. Kapitel 3.2). Da die Unterrichtseinheiten im Regelunterricht Spanisch stattfanden, war trotz des Fokus auf Mehrsprachigkeit eine gewisse sprachliche Orientierung am Spanischen vorgegeben; das bedeutet, dass auf sprachlicher Ebene nicht vollkommen frei agiert werden konnte, sondern dass bei einigen zentralen Aktivitäten ein Fokus auf das Spanische erforderlich war. Die Zweisprachigkeit der Texte gab zusätzlich jedoch einen zweiten sprachlichen Schwerpunkt vor, und zwar das Englische, das in vielen Aufgaben zusammen mit dem Spanischen gedacht, verstanden und verwendet werden sollte. Darüber hinaus erhielten die Lernenden in vielen Situationen die Möglichkeit, andere ihnen bekannte Sprachen heranzuziehen oder all ihre verfügbaren Sprachen - inklusive der verschiedenen Muttersprachen - spielerisch in den Unterricht einzubauen.

Als Unterrichtssprachen wurden entweder Spanisch oder die allen SchülerInnen gemeinsame Sprache Deutsch genutzt. Darüber hinaus wurden jedoch auch andere Sprachen - wie sich aus der Natur der Texte und der Aktivitäten logisch ergibt vor allem das Englische, teilweise aber auch Elemente aus den weiteren schulischen Fremdsprachen Französisch oder Italienisch bzw. aus anderen Muttersprachen als Deutsch - zur Kommunikation innerhalb kleinerer Gruppen eingesetzt. Vor allem für Metaphasen, in denen ein reger Austausch über die im

Barbara Hinger (Hg.), Zweite „Tagung der Fachdidaktik“ 2015.

Sprachsensibler Sach-Fach-Unterricht - Sprachen im Sprachunterricht.

(C) 2016 innsbruck university press, ISBN 978-3-903122-51-2, DOI 10.15203/3122-51-2 
Unterricht oder auch außerhalb des Unterrichts gemachten Erfahrungen sowie über persönliche Gedanken stattfand, wurde auf das Deutsche zurückgegriffen. Rückblickend lässt sich sagen, dass es sich im Sinne einer aktiven Mehrsprachigkeit hier auch angeboten hätte, die allen SchülerInnen geläufige erste Fremdsprache Englisch zu verwenden; dennoch wurde aufgrund der großen Bedeutung, die solche Metaphasen für den Lernprozess mit literarischen Texten haben (vgl. Burwitz-Melzer 2003: 427; 2006: 111), auf das Deutsche zurückgegriffen, um möglichst viele Reaktionen und Gedanken ohne eventuelle sprachliche Barrieren zu ermöglichen.

Im Folgenden wird ein Überblick über die im Unterricht durchgeführten Aktivitäten gegeben. Um eine möglichst klare Übersicht bieten zu können, werden hier nicht die eigentlichen Stundenbilder dargestellt, sondern es wird anhand einer tabellarischen Aufstellung eine Kurzbeschreibung der Hauptaktivitäten in den drei Unterrichtseinheiten gegeben.

\subsection{Unterrichtseinheit 1}

Die erste Unterrichtseinheit beinhaltet eine allgemeine Einführung ins Themengebiet und die Bearbeitung des Gedichts She. 
Tab. 1: Ablauf Unterrichtseinheit 1

\begin{tabular}{|c|c|c|c|}
\hline Aktivität & Kurzbezeichnung & $\begin{array}{l}\text { Beschreibung der } \\
\text { SchülerInnenaktivität }\end{array}$ & $\begin{array}{l}\text { verwendete } \\
\text { Sprache(n) }\end{array}$ \\
\hline A1.1 & Sprachenportrait & $\begin{array}{l}\text { Gestaltung persönlicher } \\
\text { Sprachenportraits nach Krumm \& } \\
\text { Jenkins (2001) und Vergleich mit } \\
\text { einem/r PartnerIn }\end{array}$ & $\begin{array}{l}\text { De, } \\
\text { vereinzelt } \\
\text { weitere L1 }\end{array}$ \\
\hline A1.2 & $\begin{array}{l}\text { Vorbereitung auf } \\
\text { Gedichtrezeption }\end{array}$ & $\begin{array}{l}\text { Auflistung der täglichen Aktivitäten } \\
\text { der SchülerInnen und Bewertung als } \\
\text { positiv oder negativ }\end{array}$ & Sp \\
\hline A1.3 & $\begin{array}{l}\text { Linguistische } \\
\text { Vorentlastung }\end{array}$ & $\begin{array}{l}\text { Diskussion und Erschließung der } \\
\text { im Gedicht auftretenden Verben; } \\
\text { Kategorisierung der Verben nach } \\
\text { von den SchülerInnen selbst } \\
\text { gewählten Kriterien }\end{array}$ & Sp, De \\
\hline A1.4 & $\begin{array}{l}\text { Gedichtrezeption } \\
\text { (auditiv) }\end{array}$ & $\begin{array}{l}\text { Anhören des von der Lehrperson } \\
\text { gelesenen Gedichts She und } \\
\text { Markieren der Verben als englische } \\
\text { oder spanische Verben }\end{array}$ & En, $\mathrm{Sp}$ \\
\hline A1.5 & $\begin{array}{l}\text { Charakterisierung } \\
\text { der Hauptperson }\end{array}$ & $\begin{array}{l}\text { Formulierung schriftlicher } \\
\text { Hypothesen über die Protagonistin } \\
\text { anhand eines Rasters: Alter, } \\
\text { Lebensweise, Hobbys, Beruf etc. }\end{array}$ & Sp \\
\hline A1.6 & Diskussion & $\begin{array}{l}\text { Diskussion der Ergebnisse aus A1.5, } \\
\text { Formulierung von Hypothesen über } \\
\text { die Situation der Protagonistin in } \\
\text { Partner- und Plenararbeit }\end{array}$ & De, En \\
\hline A1.7 & $\begin{array}{l}\text { Verfassen eines } \\
\text { eigenen Textes }\end{array}$ & $\begin{array}{l}\text { Verfassen eines Texts über die } \\
\text { eigene sprachliche und kulturelle } \\
\text { Situation des einzelnen Schülers/der } \\
\text { einzelnen Schülerin; verpflichtende } \\
\text { Verwendung des Spanischen und } \\
\text { zumindest einer weiteren Sprache }\end{array}$ & $\begin{array}{l}\text { De, Sp, } \\
\text { En, Fr, It, } \\
\text { weitere L1 }\end{array}$ \\
\hline
\end{tabular}

Barbara Hinger (Hg.), Zweite „Tagung der Fachdidaktik“ 2015.

Sprachsensibler Sach-Fach-Unterricht - Sprachen im Sprachunterricht.

(C) 2016 innsbruck university press, ISBN 978-3-903122-51-2, DOI 10.15203/3122-51-2 


\subsection{Unterrichtseinheit 2}

Die zweite Unterrichtseinheit beschäftigt sich mit den Gedichten Bilingual/Bilingüe und A Una Niña de Este País.

Tab. 2: Ablauf Unterrichtseinheit 2

\begin{tabular}{|c|c|c|c|}
\hline Aktivität & Kurzbezeichnung & $\begin{array}{l}\text { Beschreibung der } \\
\text { SchülerInnenaktivität }\end{array}$ & $\begin{array}{l}\text { verwendete } \\
\text { Sprache(n) }\end{array}$ \\
\hline A2.1 & Offene Erstrezeption & $\begin{array}{l}\text { Lektüre des Textes in Einzelarbeit } \\
\text { und Notieren der ersten } \\
\text { Reaktionen zu Struktur, Sprache, } \\
\text { Inhalt in Stichworten; kurze } \\
\text { Diskussion der Ergebnisse in } \\
\text { Partnerarbeit bzw. im Plenum }\end{array}$ & Sp, En, De \\
\hline A2.2 & $\begin{array}{l}\text { Auseinander-setzung } \\
\text { mit Hauptpersonen }\end{array}$ & $\begin{array}{l}\text { Formulierung von Fragen aus der } \\
\text { Perspektive einer der Personen } \\
\text { im Gedicht an die jeweils andere } \\
\text { Person in Gruppenarbeit }\end{array}$ & $\mathrm{Sp}$ \\
\hline A2.3 & Diskussion & $\begin{array}{l}\text { Diskussion der Inhalte aus A2.2; } \\
\text { Hypothesen über die Situation der } \\
\text { Protagonistin und Vergleich mit She }\end{array}$ & De \\
\hline A2.4 & $\begin{array}{l}\text { Gedichtrezeption } \\
\text { (auditiv) }\end{array}$ & $\begin{array}{l}\text { Anhören einer Lesung des } \\
\text { Gedichts A Una Niña de Este } \\
\text { País durch die Lehrperson und } \\
\text { Nacherzählung des Inhalts }\end{array}$ & Sp, En \\
\hline A2.5 & Szenische Lesung & $\begin{array}{l}\text { Mehrmaliges szenisches Lesen } \\
\text { des Textes mit verteilten Rollen in } \\
\text { Dreiergruppen }\end{array}$ & Sp, En \\
\hline A2.6 & $\begin{array}{l}\text { Charakterisierung der } \\
\text { Protagonisten }\end{array}$ & $\begin{array}{l}\text { Schriftliche Charakterisierung } \\
\text { einer der Personen im Gedicht } \\
\text { anhand gezielter Fragen und } \\
\text { anschließender Austausch mit } \\
\text { einem/r PartnerIn }\end{array}$ & $\mathrm{Sp}$ \\
\hline
\end{tabular}




\begin{tabular}{|c|c|c|c|}
\hline A2.7 & Diskussion & $\begin{array}{l}\text { Plenumsdiskussion anhand einer } \\
\text { imaginären Situation, in der die } \\
\text { SchülerInnen in ein anderes Land } \\
\text { ziehen müssen; Aufstellung entlang } \\
\text { eines gedachten Kontinuums im } \\
\text { Klassenzimmer, je nach Einstellung } \\
\text { zum Thema Beibehaltung der } \\
\text { eigenen Sprache und kulturellen } \\
\text { Zugehörigkeit oder verstärkte } \\
\text { Annäherung an Zielsprache und } \\
\text {-kultur }\end{array}$ & De \\
\hline A2.8 & $\begin{array}{l}\text { Perspektivenwechsel: } \\
\text { Verfassen eines } \\
\text { eigenen Texts }\end{array}$ & $\begin{array}{l}\text { Verfassen eines Briefs von } \\
\text { einer der beiden im Gedicht } \\
\text { vorkommenden Personen an die } \\
\text { andere auf Basis des besprochenen } \\
\text { Texts; verpflichtende Verwendung } \\
\text { des Spanischen und zumindest } \\
\text { einer weiteren Sprache }\end{array}$ & $\begin{array}{l}\text { Sp, En; tw. } \\
\text { Fr, It, De, } \\
\text { weitere L1 }\end{array}$ \\
\hline
\end{tabular}

\subsection{Unterrichtseinheit 3}

Die dritte und letzte Unterrichtseinheit beinhaltet die Bearbeitung des Gedichts Un Beso Is Not a Kiss sowie abschließende Aktivitäten, um die Erfahrungen der SchülerInnen zusammenzufassen und zu reflektieren. 
Tab. 3: Ablauf Unterrichtseinheit 3

\begin{tabular}{|c|c|c|c|}
\hline Aktivität & Kurzbezeichnung & $\begin{array}{l}\text { Beschreibung der } \\
\text { SchülerInnenaktivität }\end{array}$ & $\begin{array}{l}\text { verwendete } \\
\text { Sprache(n) }\end{array}$ \\
\hline A3.1 & $\begin{array}{l}\text { Sammlung von } \\
\text { Assoziationen }\end{array}$ & $\begin{array}{l}\text { In Gruppen: Sammlung von } \\
\text { Assoziationen zu entweder dem } \\
\text { spanischen Wort beso (dt. Kuss) } \\
\text { oder dem englischen Wort kiss auf } \\
\text { Plakaten }\end{array}$ & Sp, En \\
\hline A3.2 & $\begin{array}{l}\text { Vergleich der } \\
\text { Assoziationen }\end{array}$ & $\begin{array}{l}\text { Vergleich der Ergebnisse } \\
\text { bzgl. Gemeinsamkeiten und } \\
\text { Unterschiede, Diskussion der } \\
\text { Begriffe und unterschiedlicher } \\
\text { Konnotationen der Begriffe }\end{array}$ & Sp, En, De \\
\hline A 3.3 & $\begin{array}{l}\text { Interkomprehensives } \\
\text { Erschließen des Textes }\end{array}$ & $\begin{array}{l}\text { Lesen des Textes Un Beso Is } \\
\text { Not a Kiss und Entdecken und } \\
\text { Erschließen unbekannter Wörter } \\
\text { anhand einer interkomprehensiven } \\
\text { Wortschatztabelle unter Nutzung } \\
\text { von vorgelernten Sprachen }\end{array}$ & $\begin{array}{l}\text { Sp, De, } \\
\text { En, Fr, It, } \\
\text { weitere L1 }\end{array}$ \\
\hline A3.4 & Analyse des Textes & $\begin{array}{l}\text { Schriftliche Beantwortung und } \\
\text { Diskussion inhaltlicher Fragen } \\
\text { zum Text und zum Thema } \\
\text { Mehrsprachigkeit }\end{array}$ & Sp, De \\
\hline A 3.5 & Wortassoziationen & $\begin{array}{l}\text { Sammeln von Assoziationen zu } \\
\text { selbst gewählten Begriffspaaren } \\
\text { (z.B. noche - night, verano-summer) } \\
\text { in Spanisch und einer selbst } \\
\text { gewählten anderen Sprache }\end{array}$ & $\begin{array}{l}\text { Sp, En, } \\
\text { Fr, It, De, } \\
\text { weitere L1 }\end{array}$ \\
\hline A3.6 & Abschließende Collage & $\begin{array}{l}\text { Zusammenfassung der } \\
\text { besprochenen Texte und Thematik } \\
\text { auf einem Plakat (Zeichnungen, } \\
\text { Text, Zitate) }\end{array}$ & Sp, En \\
\hline
\end{tabular}




\section{Ausgewählte Ergebnisse}

Unter Bezugnahme auf die der Tagung der Fachdidaktik in Innsbruck grundgelegte Thematik, „Sprachsensibler Sach-Fach-Unterricht - Sprachen im Sprachunterricht", werden im Folgenden vor allem solche Ergebnisse präsentiert, welche in direktem Zusammenhang mit Sprache(n) - also im konkreten Fall mit Mehrsprachigkeit im Unterricht und mit der Zweisprachigkeit der Texte selbst - stehen. Darüber hinaus soll jedoch auch knapp die Verbindung dieser sprachlichen Aspekte mit dem soziokulturellen Hintergrund der Studie angesprochen werden. In diesem Zusammenhang soll hier noch einmal an die beiden Forschungsfragen der Studie (s. Kap. 3) erinnert werden. Einerseits stellt die Studie die Frage nach den allgemeinen Reaktionen der Lernenden auf die behandelten Texte. Dabei geht es um Denk- und Verstehensprozesse, die während der Bearbeitung der Gedichte aufkommen, sowie um Themen, die in diesem Rahmen angesprochen werden. Andererseits stellt sich darüber hinaus die Frage nach Unterschieden zwischen ein- und zweisprachig sozialisierten Jugendlichen, wenn es um die Reaktion auf die behandelten Texte geht. Diese Forschungsfragen werden nach einer Beschreibung ausgewählter Resultate (s. Kap. 6.1) mit diesen in Verbindung gebracht (s. Kap. 6.2).

Um die hier präsentierten Ergebnisse auf nachvollziehbare Weise in den Zusammenhang der Studie einbetten zu können, werden im Folgenden die aus den Daten induktiv gewonnenen Kategorien, welche auch zur Triangulation der verschiedenen Datensätze verwendet wurden, dargestellt und kurz beschrieben.

\subsection{Induktive Kategorien}

Im Rahmen der Analyse der Daten kristallisierten sich folgende zwölf Ergebniskategorien als jene Kategorien heraus, welche in den einzelnen Datensätzen das größte Gewicht erhielten: 
Tab. 4: Überblick über die induktiv gewonnenen Ergebniskategorien

\begin{tabular}{|c|c|c|c|}
\hline & Titel der Kategorie & Inhalte der Kategorie & $\begin{array}{l}\text { Präsentation } \\
\text { der Ergebnisse }\end{array}$ \\
\hline 1 & $\begin{array}{l}\text { Reaktionen auf die } \\
\text { Texte }\end{array}$ & $\begin{array}{l}\text { Aussagen der Befragten über die Texte; } \\
\text { erste Reaktionen auf spezielle Texte; } \\
\text { Erinnerungen an Eigenschaften der } \\
\text { Texte; allgemeine Aussagen zur Form } \\
\text { der Gedichte; Aussagen zu positiver oder } \\
\text { negativer Wirkung der Texte auf die } \\
\text { TeilnehmerInnen }\end{array}$ & s. Kap. 6.1 .1 \\
\hline 2 & $\begin{array}{l}\text { Sprachliches } \\
\text { Verständnis der Texte }\end{array}$ & $\begin{array}{l}\text { initiales Verständnis der sprachlichen } \\
\text { Oberflächenstruktur der Texte; eventuelle } \\
\text { Schwierigkeiten; Prozesse bei der } \\
\text { Textlektüre }\end{array}$ & nicht inkludiert \\
\hline 3 & $\begin{array}{l}\text { Wahrnehmung der } \\
\text { Zweisprachigkeit in } \\
\text { den Gedichten }\end{array}$ & $\begin{array}{l}\text { positive oder negative Reaktionen auf die } \\
\text { Zweisprachigkeit in den Texten }\end{array}$ & s. Kap. 6.1 .2 \\
\hline 4 & $\begin{array}{l}\text { (Mehrsprachiger) } \\
\text { Schreibprozess }\end{array}$ & $\begin{array}{l}\text { Aussagen über Schwierigkeiten oder } \\
\text { Erfolgserlebnisse im Rahmen der } \\
\text { Verwendung von mehr als einer Sprache } \\
\text { im Schreibprozess }\end{array}$ & s. Kap. 6.1 .4 \\
\hline 5 & $\begin{array}{l}\text { Sprachverwendung in } \\
\text { den selbst verfassten } \\
\text { Texten }\end{array}$ & $\begin{array}{l}\text { Thematisierung der Verwendung } \\
\text { von verschiedenen Sprachen in den } \\
\text { Texten selbst bzw. in den Aussagen der } \\
\text { TeilnehmerInnen }\end{array}$ & s. Kap. 6.1 .4 \\
\hline 6 & $\begin{array}{l}\text { Auseinandersetzung } \\
\text { mit den Charakteren } \\
\text { und Verständnis ihrer } \\
\text { Situation }\end{array}$ & $\begin{array}{l}\text { Aussagen über die Charaktere und deren } \\
\text { Situation sowie über deren Beziehung } \\
\text { zueinander }\end{array}$ & nicht inkludiert \\
\hline 7 & $\begin{array}{l}\text { Verbindung } \\
\text { mit eigener } \\
\text { Erfahrungswelt }\end{array}$ & $\begin{array}{l}\text { persönliche Verbindung, welche die } \\
\text { TeilnehmerInnen mit den Texten } \\
\text { herstellen }\end{array}$ & s. Kap. 6.1 .5 \\
\hline
\end{tabular}

Barbara Hinger (Hg.), Zweite „Tagung der Fachdidaktik“ 2015.

Sprachsensibler Sach-Fach-Unterricht - Sprachen im Sprachunterricht.

(C) 2016 innsbruck university press, ISBN 978-3-903122-51-2, DOI 10.15203/3122-51-2 


\begin{tabular}{|c|c|c|c|}
\hline 8 & $\begin{array}{l}\text { Übergreifende } \\
\text { Thematik der Texte }\end{array}$ & $\begin{array}{l}\text { Ansichten der TeilnehmerInnen zur } \\
\text { Kernaussage der Texte }\end{array}$ & nicht inkludiert \\
\hline 9 & $\begin{array}{l}\text { Eigene Wahrnehmung } \\
\text { als ein- oder } \\
\text { mehrsprachige Person }\end{array}$ & $\begin{array}{l}\text { Aussagen der TeilnehmerInnen zu ihrer } \\
\text { eigenen Sprachsituation bzw. -identität }\end{array}$ & nicht inkludiert \\
\hline 10 & $\begin{array}{l}\text { Ansichten zum } \\
\text { mehrsprachigen } \\
\text { Unterricht }\end{array}$ & $\begin{array}{l}\text { Aussagen zum sprachenübergreifenden } \\
\text { Arbeiten in einer Unterrichtseinheit; } \\
\text { positive wie negative Aspekte sowie die } \\
\text { individuellen Theorien der Lernenden } \\
\text { in Bezug auf Vor- und Nachteile des } \\
\text { sprachenübergreifenden Ansatzes }\end{array}$ & s. Kap. 6.1 .3 \\
\hline 11 & $\begin{array}{l}\text { (Metasprachliche) } \\
\text { Überlegungen } \\
\text { zu Sprachen und } \\
\text { Mehrsprachigkeit }\end{array}$ & $\begin{array}{l}\text { Ideen und Ansätze der TeilnehmerInnen } \\
\text { zum Thema Sprache und } \\
\text { Mehrsprachigkeit; eigene Theorien } \\
\text { zum Sprachenlernen oder zum } \\
\text { mehrsprachigen Lernen }\end{array}$ & nicht inkludiert \\
\hline 12 & $\begin{array}{l}\text { Eignung der Texte } \\
\text { und persönliche } \\
\text { Präferenz }\end{array}$ & $\begin{array}{l}\text { Aussagen der Lernenden über die } \\
\text { Eignung der einzelnen Texte für den } \\
\text { Einsatz im Unterricht; Präferenz } \\
\text { einzelner TeilnehmerInnen für bestimmte } \\
\text { Texte }\end{array}$ & nicht inkludiert \\
\hline
\end{tabular}

Um dem Thema des vorliegenden Bandes Rechnung zu tragen, werden, wie bereits erwähnt, für die folgende Ergebnispräsentation beinahe ausschließlich solche Kategorien verwendet, welche mit Sprache(n) - also im konkreten Fall mit Mehrsprachigkeit im Unterricht und der Zweisprachigkeit der Texte - zu tun haben; teilweise werden dabei auch mehr als eine Kategorie zusammengefasst, um einer zu extensiven Beschreibung vorzubeugen. Eine der präsentierten Kategorien (s. Kap. 6.1.5) stellt eine Ausnahme dar, da sie sich auf den soziokulturellen Hintergrund der TeilnehmerInnen und nicht auf ihre sprachliche Verortung bezieht; diese Kategorie wurde aufgrund ihrer zentralen Bedeutung für die Forschungsfragen inkludiert. Ein paar wenige Kategorien werden hier aufgrund der hohen 
Spezifität ihrer Ergebnisse nicht inkludiert; stattdessen konzentrieren sich die folgenden Absätze auf jene Kategorien, welche allgemeinere Aussagen zulassen.

\subsubsection{Erhöhte Akzeptanz eigener und fremder Mehrsprachigkeit}

Was sich in der Analyse der Daten durchgehend beobachten lässt, sowohl bei einals auch bei zweisprachig sozialisierten TeilnehmerInnen, ist ein grundlegendes Bewusstwerden über die eigenen Einstellungen und Gefühle gegenüber einzelnen Sprachen und kulturellen Einflüssen, welche die SchülerInnen in ihrer individuellen Verortung prägen. Durch die Verwendung aller vorhandenen Sprachen im Klassenzimmer, durch die Lektüre und Bearbeitung von authentischen zweisprachigen Texten sowie durch den Einsatz von sprachenübergreifenden Strategien im Unterricht konnte die Wahrnehmung und in Verbindung damit auch die Anerkennung der verschiedenen Sprachen, die in jedem/r einzelnen SchülerIn präsent sind, sowie der sprachlichen Mischformen im täglichen Sprachgebrauch von Menschen mit Migrationshintergrund geweckt und gestärkt werden. Besonders dem Prozess des mehrsprachigen Schreibens, wie er im Rahmen der Studie eingesetzt wurde, kommt im Zusammenhang mit dieser Bewusstwerdung eine wichtige Rolle zu: Die TeilnehmerInnen brachten vor allem im Falle des ersten selbst verfassten Gedichts in Reaktion auf Elizondos She ihre persönlichen Einstellungen und Gefühle gegenüber ihren verschiedenen Sprachen zum Ausdruck, was in Kapitel 6.1.4 ausführlicher diskutiert wird.

Zusätzlich konnte im Rahmen der Unterrichtsaktivitäten das Bewusstsein der SchülerInnen dafür gestärkt werden, dass Sprachen nicht nur Unterschiede, sondern auch Gemeinsamkeiten aufweisen. Vor allem durch das Sammeln von ähnlichen Begriffen in verschiedenen Sprachen im Rahmen der Bearbeitung von Un Beso Is Not a Kiss in der letzten Unterrichtseinheit wurden sich die SchülerInnen darüber bewusst, inwieweit auch vermeintlich sehr fremde Sprachen beispielsweise mit der eigenen Muttersprache zusammenhängen. Da sich die SchülerInnen nicht nur ihrer Schulfremdsprachen bedienten, sondern auch Interesse an den Muttersprachen ihrer MitschülerInnen zeigten - beispielsweise indem sie diese um Übersetzungen in ihnen bisher fremde Sprachen baten - konnten hier ungeahnte Erschließungs- und Begegnungspotenziale freigelegt werden.

Barbara Hinger (Hg.), Zweite „Tagung der Fachdidaktik“ 2015.

Sprachsensibler Sach-Fach-Unterricht - Sprachen im Sprachunterricht.

(C) 2016 innsbruck university press, ISBN 978-3-903122-51-2, DOI 10.15203/3122-51-2 


\subsubsection{Reaktionen auf die Zweisprachigkeit der Texte}

In diesem Bereich lässt sich - im Gegensatz zu anderen untersuchten Aspekten ein Unterschied zwischen ein- und mehrsprachig sozialisierten TeilnehmerInnen festmachen. Vor allem die lebensweltlich einsprachigen SchülerInnen reagierten beim Erstkontakt mit Elizondos She - dem ersten bearbeiteten Text in der Unterrichtsreihe - erstaunt, teilweise sogar irritiert oder ablehnend auf die Verwendung von mehr als einer Sprache innerhalb eines zusammenhängenden Texts; dies hing einerseits mit der Neuartigkeit einer solchen übergreifenden Sprachverwendung zusammen, andererseits jedoch auch mit dem höheren Schwierigkeitsgrad in Bezug auf das Textverständnis, wie es von einzelnen lebensweltlich einsprachigen TeilnehmerInnen thematisiert wurde. Interessanterweise verringerte sich dieses Gefühl der Ablehnung oder Irritation im Laufe der Unterrichtseinheiten bei allen TeilnehmerInnen, als sie mit dieser Art der Sprachverwendung vertrauter wurden (vgl. Mayr 2014). Die lebensweltlich zweisprachigen SchülerInnen erwähnten in diesem Zusammenhang hingegen häufig ihre Vertrautheit mit zweisprachiger Sprachproduktion - beispielsweise beim Verfassen zweisprachiger Textnachrichten am Smartphone - oder thematisierten diese überhaupt nicht. Dies ist vermutlich darauf zurückzuführen, dass ein zweisprachiges Aufwachsen die simultane Verwendung von mehr als einer Sprache in bestimmten Situationen automatisch und auf natürliche Weise mit sich bringt und dass für zweisprachig sozialisierte SprecherInnen folglich ein Wechsel zwischen verschiedenen Sprachen in ihrem alltäglichen Sprachgebrauch als nicht ungewöhnlich, sondern als üblich gilt (u.a. Gafaranga 2007: 3; Wei 2011: 370).

\subsubsection{Einstellungen zum mehrsprachigen Unterricht}

Da sich die hier beschriebene Studie unter anderem auf der Idee einer mehrsprachigen Unterrichtspraxis begründet, wurde im Rahmen der Datenerhebung und -analyse auch die Meinung der TeilnehmerInnen zu einem sprachenübergreifenden Unterricht erhoben. Wie Gorter \& Cenoz (2011: 444) betonen, sollte die Unterrichtspraxis im sprachenübergreifenden Fremdsprachenunterricht diejenigen mehrsprachigen Strategien als Ausgangspunkt nehmen, welche die 
SchülerInnen selbst verwenden. In der hier beschriebenen Studie wurde im Zusammenhang mit solchen mehrsprachigen Strategien sowohl auf die typischerweise von lebensweltlich zweisprachigen Jugendlichen verwendeten Strategien wie code-switching oder translanguaging als auch auf die stärker kognitiven Techniken der Interkomprehensionsdidaktik, welche vor allem das Erlernen von Schulfremdsprachen fokussiert, eingegangen. Anzumerken ist dabei vor allem, dass das mehrsprachige Arbeiten vom Großteil der TeilnehmerInnen sehr positiv aufgenommen wurde, zum Teil reagierten die Lernenden geradezu begeistert darauf, und dass eventuelle als solche empfundene Nachteile dieser Arbeitsweise durch die genannten Vorteile wieder aufgewogen oder - nach dem Empfinden der TeilnehmerInnen - sogar übertroffen wurden.

Sowohl im Falle der lebensweltlich einsprachigen als auch der lebensweltlich zweisprachigen TeilnehmerInnen zeigt sich ein klares Bild, wenn es um die Vorbzw. Nachteile eines sprachenübergreifenden Ansatzes geht: Der Großteil aller SchülerInnen erwähnt als potenziellen Nachteil die Interferenzen zwischen den verwendeten Sprachen, wobei dies im Empfinden der meisten TeilnehmerInnen auf einander sehr ähnliche Sprachen beschränkt bleibt. Die Problematik der sogenannten ,falschen Freunde sorgt auch in der wissenschaftlichen Diskussion um eine Mehrsprachigkeitsdidaktik - vor allem auf Grundlage der Kontrastivhypothese - für Bedenken; meist kommen ExpertInnen jedoch zu dem Schluss, dass diese Gefahr geringer ist, als gemeinhin vermutet wird, und dass die Vorteile, welche in der Ähnlichkeit der Sprachen liegen, bei weitem überwiegen (vgl. z.B. Klein 2004; Bär 2009).

Ein weiterer Nachteil, welcher teilweise von den Lernenden erwähnt wird, ist der von ihnen wahrgenommene kognitive Mehraufwand bei der gleichzeitigen Verwendung mehrerer Sprachen. Dieser Aspekt wird in der Diskussion um eine mehrsprachige Didaktik äußerst selten erwähnt, es finden sich jedoch einzelne Hinweise darauf, dass solche Überlegungen ihre Berechtigung haben (z.B. Mertens 2006; Delanoy 2014). Delanoy (2014) weist demzufolge auf die Notwendigkeit hin, entsprechende Texte zu verwenden, die für die Lernenden kein zu großes sprachliches Problem darstellen. In der hier beschriebenen Studie bereitete das sprachliche Verständnis nach Aussage der TeilnehmerInnen, wohl auch aufgrund der auf ihren Lernstand zugeschnittenen Textauswahl, weder in der

Barbara Hinger (Hg.), Zweite „Tagung der Fachdidaktik“ 2015.

Sprachsensibler Sach-Fach-Unterricht - Sprachen im Sprachunterricht.

(C) 2016 innsbruck university press, ISBN 978-3-903122-51-2, DOI 10.15203/3122-51-2 
lebensweltlich einsprachigen noch in der lebensweltlich zweisprachigen Gruppe Schwierigkeiten. Trotz der hier beschriebenen Nachteile schlussfolgern insgesamt beide Lerngruppen beinahe einstimmig, dass ein sprachenübergreifender Ansatz im Kontext des Fremdsprachenunterrichts hauptsächlich positive Konsequenzen mit sich bringt und dass diese die oben erwähnten Nachteile deutlich übertreffen. Damit gehen sie sowohl mit VertreterInnen einer rezeptiv ausgerichteten Mehrsprachigkeitsdidaktik (u.a. Meißner 2008; Meißner \& Reinfried 1998; Hufeisen \& Neuner 2003; Bär 2008, 2009) als auch mit VerfechterInnen eines weiter gefassten Konzepts mehrsprachigen Unterrichts (u.a. García et al. 2006; López-Gopar et al. 2013; Schecter \& Cummins 2003; van der Walt 2013; Allgäuer et al. 2014) konform.

Wenn es um die genannten Vorteile geht, dann argumentieren die lebensweltlich ein- und zweisprachigen TeilnehmerInnen nur teilweise in eine ähnliche Richtung. Ein Faktor, welcher in beiden Gruppen zur Sprache kommt, ist die Übertragbarkeit von Wortschatzkenntnissen und von Strukturen von einer in die andere Sprache, also der positive Transfer von sprachlichen Elementen zwischen verschiedenen Sprachen. Dieser ist zentrales Element in interkomprehensiv geprägten Konzepten der Mehrsprachigkeitsdidaktik und bildet eine wichtige Grundlage für Theoriebildung und Forschung zur Interkomprehension (vgl. u.a. Meißner 2008; Meißner \& Reinfried 1998; Hufeisen \& Neuner 2003; Bär 2008).

Über diesen zentralen Faktor hinaus wird auch die erhöhte Motivation, welche das sprachenübergreifende Arbeiten mit sich bringen kann, von beiden Gruppen angesprochen. Die Begründungszusammenhänge klaffen hier jedoch auseinander: Die lebensweltlich einsprachigen TeilnehmerInnen argumentieren mit der abwechslungsreicheren Unterrichtsgestaltung, welche mit der Verwendung mehrerer Sprachen ihres Erachtens einhergeht und damit ihre Motivation erhöht; die zweisprachig sozialisierten Teilnehmerinnen hingegen bringen eine persönliche Ebene ins Spiel, welche die in den Texten vorkommende und beschriebene Mehrsprachigkeit und -kulturalität auf ihre eigene Identität umlegt und damit ihr Interesse an den präsentierten Texten und an den damit verbundenen Aktivitäten förderte. Darüber hinaus sehen gerade die zweisprachig sozialisierten Teilnehmerinnen die Vorteile eines sprachenübergreifenden Ansatzes stark auf der Ebene des Sprachbewusstseins, welches ihres Erachtens mit der Auseinandersetzung

Barbara Hinger (Hg.), Zweite „Tagung der Fachdidaktik“ 2015.

Sprachsensibler Sach-Fach-Unterricht - Sprachen im Sprachunterricht.

(C) 2016 innsbruck university press, ISBN 978-3-903122-51-2, DOI 10.15203/3122-51-2 
mit mehrsprachigen Texten einhergeht. Hier wird unter anderem die Sensibilisierung für Unterschiede und Ähnlichkeiten zwischen verschiedenen Sprachen erwähnt. Dieser Faktor wird von den einsprachig sozialisierten TeilnehmerInnen der Studie hingegen nicht thematisiert.

Als weiterer Vorteil wird teilweise von beiden Gruppen die Automatisierung von Sprachformen thematisiert, gemeint ist damit die Förderung eines schnellen und problemlosen Wechsels zwischen verschiedenen Sprachen. Christ (2004: 31) spricht in diesem Zusammenhang von einer Befähigung zum „Handeln in mehreren Sprachen“, zur Kommunikation über die Sprachen hinweg, in der die Lernenden sich über Möglichkeiten und Grenzen ihrer Kommunikationsfähigkeit bewusst werden können. Auch Strategien zur Lösung von Problemen der sprachenübergreifenden Kommunikation können hier mit einfließen. Die SchülerInnen sprechen also sehr konkret ein Ziel des sprachenübergreifenden Fremdsprachenunterrichts an.

\subsubsection{Mehrsprachiger Schreibprozess}

Zahlreiche ExpertInnen (u.a. Blell 2009, Cahnmann 2006, Cruz 2011, Elsner 2012) schreiben dem Prozess des mehrsprachigen Schreibens positive Auswirkungen auf die kreative Ausdrucksfähigkeit sowie auf die Reflexionsfähigkeit der Lernenden in Bezug auf ihre eigene Identität zu. In der hier beschriebenen Studie wurden diese Effekte vor allem im Rahmen des ersten selbst verfassten Gedichts deutlich, in dem die TeilnehmerInnen zum Großteil ihre persönlichen Einstellungen und Gefühle gegenüber ihren verschiedenen Sprachen beschrieben. Auch wenn keine/r der TeilnehmerInnen explizit die Möglichkeit erwähnt, sich selbst als Person besser zum Ausdruck bringen zu können, wenn mehrere Sprachen involviert sind; dennoch wird in beiden Gruppen in vielen Texten und Stimulated recall-Kommentaren klar, dass die Verwendung verschiedener Sprachen eine Reflexion über das eigene Sprachverhalten auslöste. Hier bestätigen die Ergebnisse der Studie also die oben präsentierten ExpertInnenmeinungen zur Gänze.

In Bezug auf den eigentlichen Schreibprozess erwähnen sowohl lebensweltlich einsprachige als auch zweisprachige TeilnehmerInnen einerseits Freude an der

Barbara Hinger (Hg.), Zweite „Tagung der Fachdidaktik“ 2015.

Sprachsensibler Sach-Fach-Unterricht - Sprachen im Sprachunterricht.

(C) 2016 innsbruck university press, ISBN 978-3-903122-51-2, DOI 10.15203/3122-51-2 
gleichzeitigen Verwendung mehrerer Sprachen sowie eine damit einhergehende Vereinfachung des Verfassens des Textes, benennen andererseits jedoch auch auftretende Schwierigkeiten, wobei sich diese hauptsächlich auf den Gebrauch des Spanischen und nicht prinzipiell auf die gleichzeitige Verwendung mehrerer Sprachen beziehen. Grundsätzlich herrschte in beiden Gruppen eine positive Einstellung dem Schreiben in verschiedenen Sprachen gegenüber vor. Dies wird auch bei einem Blick auf die selbst verfassten Texte bestätigt: Vor allem bei dem Gedicht in Reaktion auf Elizondos She verwenden die Schülerinnen auffallend viele verschiedene Sprachen und bauen in vereinzelten Fällen auch Sprachen ein, welche nur einen marginalen Platz in ihrem lebensweltlichen Sprachgefüge einnehmen. Hier stimmen die Ergebnisse der Studie mit Aussagen von Cruz (2011) überein, der die Verwendung von mehr als einer Sprache im kreativen Schreiben als künstlerisches Werkzeug zur Produktion von Kunstwerken ins Treffen führt und beschreibt.

Im Gegensatz dazu erwähnt Busch (2011) als negativen Faktor des mehrsprachigen Schreibens die Tatsache, dass die Möglichkeit zur Verwendung verschiedener Sprachen die SchülerInnen dazu verleitet, ausschließlich oder hauptsächlich die Sprache einzusetzen, die ihnen am geläufigsten ist. In Bezug auf den ersten selbst verfassten Text wird diese ,Gefahr ${ }^{6}$ in der hier beschriebenen Studie nur von einer Teilnehmerin explizit bestätigt, die erwähnt, hauptsächlich diejenigen Sprachen verwendet zu haben, welche sie am besten beherrscht; der von Busch vorgebrachte Einwand kann im Fall des ersten Texts also nicht bestätigt werden. Hinsichtlich des zweiten selbst verfassten Texts liegt jedoch durchaus Grund zu einer genaueren Betrachtung vor: In beiden Gruppen verwendeten einige TeilnehmerInnen eine Sprachform, welche nicht mit dem Ausgangstext kongruent ist; dabei handelt es sich um eine zu häufige bzw. ausschließliche Verwendung des Englischen; dies wird meist mit der fehlenden Sprachkompetenz im Spanischen begründet. In beiden Gruppen lässt sich im Falle des zweiten Texts also eine deutliche Parallele zu Buschs (2011) Bedenken ziehen.

Barbara Hinger (Hg.), Zweite „Tagung der Fachdidaktik“ 2015.

Sprachsensibler Sach-Fach-Unterricht - Sprachen im Sprachunterricht.

(C) 2016 innsbruck university press, ISBN 978-3-903122-51-2, DOI 10.15203/3122-51-2 


\subsubsection{Transfer auf die eigene Lebensrealität}

Obwohl der in der Studie eingehend untersuchte Aspekt des Transfers auf die eigene Lebenswirklichkeit nicht direkt mit Sprachverwendung im Unterricht zu tun hat, soll er hier aufgrund seiner zentralen Stellung innerhalb der Untersuchung erwähnt und kurz beschrieben werden. Wie in vielen anderen Bereichen lässt sich auch in diesem Bereich keine Unterscheidung zwischen lebensweltlich ein- und zweisprachigen TeilnehmerInnen festmachen; trotzdem oder vielleicht gerade deshalb stellen sich die Ergebnisse in diesem Bereich als interessant und aufschlussreich dar. Das Aufstellen bzw. Erkennen von Verbindungen zwischen literarischen Texten und der eigenen Lebenswelt beschreibt Burwitz-Melzer (2003: 159) in ihrem Katalog von Lernertätigkeiten im interkulturellen Lernen anhand von literarischen Texten wie folgt: „Die Jugendlichen [...] erwähnen eigene Erfahrungen als Folie zu dem im Text dargestellten Konflikt." Wie Bredella (u.a. 2010: XXIV) betont, tritt der/die LeserIn beim Eintauchen in einen literarischen Text in einen Dialog mit den Perspektiven, die ihm/ihr im Text präsentiert werden; dies geschieht durch Einnahme der Innen- und Reflexion durch die Außenperspektive. Teilweise kommt es hier zur Bewusstwerdung über Unterschiede, aber auch zum Erkennen von Parallelen mit der eigenen Lebenserfahrung.

In der hier beschriebenen Studie wurden von den TeilnehmerInnen vor allem Parallelen thematisiert, wobei sich wiederum - wie bereits erwähnt - bei den ein- und zweisprachig sozialisierten TeilnehmerInnen ein nicht durchgängig kohärentes Bild ergibt: Etwas mehr als die Hälfte der lebensweltlich einsprachigen TeilnehmerInnen sieht laut eigener Aussage keine Verbindung zwischen dem Inhalt der bearbeiteten Texte und ihrer persönlichen Lebenssituation; die restlichen TeilnehmerInnen stellen entweder Verbindungen zu ihrer eigenen Situation oder zu der von Menschen in ihrem Umfeld auf. Im Falle der lebensweltlich zweisprachigen Schülerinnen ergibt sich ein ähnliches Bild: Die Hälfte der TeilnehmerInnen zieht von sich aus eine Parallele zum eigenen Leben, während die restlichen TeilnehmerInnen keine Parallele zu ihrer eigenen Situation erkennen.

Ein erfolgreicher Transfer der zwischensprachlichen und -kulturellen Erkenntnisse, welche aus der Auseinandersetzung mit den sprachen- und kulturenüber-

Barbara Hinger (Hg.), Zweite „Tagung der Fachdidaktik“ 2015.

Sprachsensibler Sach-Fach-Unterricht - Sprachen im Sprachunterricht.

(C) 2016 innsbruck university press, ISBN 978-3-903122-51-2, DOI 10.15203/3122-51-2 
greifenden Texten resultieren, auf eigene Erfahrungen kann also - wenn auch meist in unterschiedlichen Kontexten - sowohl bei lebensweltlich zweisprachigen als auch bei lebensweltlich einsprachigen Jugendlichen stattfinden und bleibt nicht auf jene beschränkt, welche selbst in einer ähnlichen Situation aufgewachsen sind. Ebenfalls findet dieser Transfer automatisch bei zweisprachig sozialisierten Jugendlichen statt. Dies kann unter anderem auf Sexls (2003: 277) Beobachtung zurückzuführen sein, dass die Herstellung von Verbindungen zwischen literarischen Texten und der Lebensrealität des Lesepublikums nicht immer von selbst erfolgt, sondern vielmehr durch teilweise „hartnäckiges Nachfragen“ von Seiten des Forschers/der Forscherin zutage tritt; in der hier beschriebenen Untersuchung wurde von einem solchen Nachfragen jedoch abgesehen, um die SchülerInnen in ihrer Reaktion nicht unnötig zu beeinträchtigen.

\subsection{Reflexion der Ergebnisse auf Basis der Forschungsfragen}

Der Darstellung der Ergebnisse soll nun eine Verbindung zu den eigentlichen Forschungsfragen folgen, welche eine engere Einbettung in den Kontext der Studie zulässt. Bezüglich der ersten, sehr allgemein gehaltenen Forschungsfrage, welche auf das Sichtbarmachen von Denk- und Verstehensprozessen der Lernenden bei der Bearbeitung von mehrsprachigen literarischen Texten abzielt, lässt sich - wie erwartet und auch beabsichtigt - eine sehr große Bandbreite an verschiedenen Resultaten beschreiben. Sowohl in Bezug auf die Rezeption als auch auf die Produktion von mehrsprachigen Texten sowie auf die Ansichten der TeilnehmerInnen zu Themen der persönlichen Mehrsprachigkeit und des mehrsprachigen Unterrichtens lassen sich hier individuell sehr verschiedene Aussagen bzw. Ansichten beobachten und beschreiben (vgl. Mayr, i.Dr.).

In Bezug auf die zweite Forschungsfrage nach möglichen Unterschieden zwischen ein- und zweisprachig sozialisierten Jugendlichen in ihrer Reaktion auf die Texte lässt sich Folgendes feststellen: Im Verlauf der Datenanalyse wurde zunehmend klar, dass sich - im Gegensatz zu anfänglichen Überlegungen von Seiten der Forscherin, welche Unterschiede zwischen lebensweltlich einsprachigen und lebensweltlich zweisprachigen SchülerInnen in Erwartung stellten - nur in wenigen und ganz bestimmten Aspekten eine Unterscheidung zwischen die-

Barbara Hinger (Hg.), Zweite „Tagung der Fachdidaktik“ 2015.

Sprachsensibler Sach-Fach-Unterricht - Sprachen im Sprachunterricht.

(C) 2016 innsbruck university press, ISBN 978-3-903122-51-2, DOI 10.15203/3122-51-2 
sen beiden Gruppen zeigt. Vielmehr legen die Ergebnisse den Schluss nahe, dass eine mögliche Differenzierung zwischen den TeilnehmerInnen nicht vorrangig auf deren lebensweltliche Sprach- und Kultursituation zurückzuführen ist, sondern dass sich ihre Denkprozesse und Herangehensweisen aufgrund diverser individueller Charakteristika unterscheiden, wie sie in Kapitel 7 kurz angerissen werden. In vielen Aspekten, welche im Rahmen der Analyse untersucht wurden (beispielsweise in Bezug auf Empathiefähigkeit der TeilnehmerInnen oder auf das Herstellen von Verbindungen zwischen dem eigenen Leben und den bearbeiteten Texten), finden sich sowohl lebensweltlich ein- als auch zweisprachige TeilnehmerInnen, welche in den untersuchten Kategorien eine ähnliche Tendenz zeigen, jedoch auch solche, die dieser Tendenz widersprechen; deshalb lassen es die Ergebnisse der vorliegenden Studie nicht zu, von einer Unterscheidung nach lebensweltlichem Sprach- und Kulturhintergrund zu sprechen.

\section{Konklusionen}

Als Resümee bleibt anzumerken, dass das mehrsprachige Arbeiten im Spanischunterricht insgesamt äußerst positiv und von einigen TeilnehmerInnen sogar mit Enthusiasmus aufgenommen wurde. Die Freude am gleichzeitigen Verwenden aller den LernerInnen zur Verfügung stehenden Sprachen und am spielerischen Umgang mit diesen Sprachen, wie er auch in den verwendeten literarischen Texten praktiziert wird, wurde vor allem in den Abschlussinterviews von vielen TeilnehmerInnen kommentiert und als besonders positiv hervorgehoben. Mit solchen und ähnlichen Unterrichtsprojekten kann, wie eben in Kapitel 6 beschrieben, ein wichtiger Schritt in Richtung eines bewussten und wertschätzenden Umgangs mit der eigenen Mehrsprachigkeit sowie mit den sprachlichen Fähigkeiten anderer gesetzt werden.

In Bezug auf die Frage nach möglichen Unterschieden zwischen ein- und zweisprachig sozialisierten Jugendlichen in der Reaktion auf die Texte, lässt sich, wie bereits erwähnt, Folgendes feststellen: Eventuelle Unterschiede, die sich in der Bearbeitung und Reaktion auf die behandelten Texte ergeben, sind nicht vordergründig auf den lebensweltlichen Sprach- und Kulturhintergrund der

Barbara Hinger (Hg.), Zweite „Tagung der Fachdidaktik“ 2015.

Sprachsensibler Sach-Fach-Unterricht - Sprachen im Sprachunterricht.

(C) 2016 innsbruck university press, ISBN 978-3-903122-51-2, DOI 10.15203/3122-51-2 
TeilnehmerInnen zurückzuführen, sondern dürften von verschiedenen anderen Faktoren abhängig sein. Unter anderem wäre es denkbar, dass Unterschiede im Bildungshintergrund des Elternhauses oder in der grundsätzlichen Interessenslage (z.B. Interesse für Literatur oder Lesen im Allgemeinen) für differierende Reaktionen verantwortlich sind. Ein weiterer Faktor könnte im Geschlecht der LeserInnen liegen sowie in der Schulform, welche sie besuchen. Es ließen sich in diesem Zusammenhang zahlreiche weitere untersuchungswürdige Aspekte andenken; klar wird in jedem Fall, dass die unterschiedlichen Reaktionen und Einstellungen der TeilnehmerInnen der hier beschriebenen Studie von einem komplexen Gefüge an Faktoren abhängig sind und dass eine kausale Rückführung auf einen einzelnen Aspekt nur eine sehr eingeschränkte Sicht auf den untersuchten Forschungsbereich zuließe. Es verhält sich hier wohl ähnlich wie bei Ettes (2005: 15) Beschreibung von Literaturen ohne festen Wohnsitz: solche Texte einzig und allein über den Faktor der Migration zu definieren wäre laut Ette deutlich zu kurz gegriffen, vielmehr müssen andere Faktoren bei ihrer Beschreibung und Analyse ebenso in den Blick genommen werden.

\section{Literatur}

Alarcón, F. X. (1989). Un Beso Is Not a Kiss. In J. Palley (Hrsg.), Best New Chicano Literature 1989 (19). Tempe, AZ: Bilingual Press/Editorial Bilingüe.

Allgäuer-Hackl, E., Jessner-Schmid, U. \& Oberhofer, K. (2013). Mehrsprachige Entwicklung - Was sagt die Forschung? In G. Gombos (Hrsg.), Mehrsprachigkeit grenzüuberschreitend. Modelle, Konzepte, Erfahrungen (68-87). Klagenfurt, Meran: Drava, Alpha Beta.

Bär, M. (2008). Intercomprensión en la clase de español. Fomento de la conciencia lingüística y de la competencia de aprender a aprender. In U. Vences (Hrsg.), Sprache - Literatur - Kultur. Vernetzung im Spanischunterricht (26-40). Berlin: edition tranvía - Verlag Walter Frey.

Bär, M. (2009). Förderung von Mebrsprachigkeit und Lernkompetenæ. Fallstudien zu Interkomprehensionsunterricht mit Schülern der Klassen 8 bis 10. Tübingen: Narr.

Blell, G. (2009). Cross-Perspectives. Verknüpfungsmöglichkeiten Englisch-Spanisch am Beispiel Sandra Cisneros' Caramelo. Praxis Fremdsprachenunterricht, 6 (2), 19-23. 
Blell, G. (2012). Literarisches Lernen und Sprach(en)lernen im Fremdsprachenunterricht. In E. Burwitz-Melzer, F. G. Königs \& H.-J. Krumm (Hrsg.), Sprachenbewusstheit im Fremdsprachenunterricht. Arbeitsmappe der 32. Frübjabrskonferenz zur Erforschung des Fremdsprachenunterrichts (9-18). Tübingen: Narr Verlag.

Bredella, L. (2010). Das Verstehen des Anderen. Kulturwissenschaftliche und literaturdidaktische Studien. Tübingen: Narr.

Bredella, L. \& Burwitz-Melzer, E. (2004). Rezeptionsästhetische Literaturdidaktik. Mit Beispielen aus dem Fremdsprachenunterricht Englisch. Tübingen: Narr.

Bruce-Novoa, J. D. (1982). Chicano poetry. A response to chaos (1. Aufl.). Austin: University of Texas Press.

Burwitz-Melzer, E. (2003). Allmäbliche Annäherungen. Fiktionale Texte im interkulturellen Fremdsprachenunterricht der Sekundarstufe I. Tübingen: Narr.

Burwitz-Melzer, E. (2006). Interkulturelles und sprachliches Lernen mit fremdsprachlichen literarischen Texten: Zwei zentrale Elemente eines neuen Lesekompetenzmodells. Fremdsprachen Lebren und Lernen (C. Gnutzmann, F. G. Königs \& E. Zöfgen (Hrsg.), Zur Theorie und Praxis des Sprachunterrichts an Hochschulen. Themenschwerpunkt: Sprachdidaktik - interkulturell), 104-120.

Busch, B. (2011). Trends and innovative practices in multilingual education in Europe. An overview. International Review of Education 57 (5-6), 541-549.

Cahnmann, M. (2006). Bilingual Poetry as ScholARTistry in the Language Arts Classroom. Language Arts, 83 (4), 342-352.

Cenoz, J. \& Genesee, F. (1998). Beyond bilingualism. Multilingualism and multilingual education. Clevedon, England: Multilingual Matters.

Cenoz, J. \& Gorter D. (2010). The Diversity of Multilingualism in Education. International Journal of the Sociology of Language 205, 37-53.

Christ, H. (2004). Didaktik der Mehrsprachigkeit im Rahmen der Fremdsprachendidaktik. In K.-R. Bausch, F. G. Königs \& H.-J. Krumm (Hrsg.), Mebrspracbigkeit im Fokus. Arbeitspapiere der 24. Frühjahrskonferenz zur Erforschung des Fremdsprachenunterrichts (30-38). Tübingen: Narr.

Cruz, I. R. (2011). Writing with Two Languages. TEXT, 15 (Special Issue Website Series Number 10: Creative Writing in the Asia-Pacific Region), 1-13. Verfügbar unter: http://www.textjournal.com.au/speciss/issue10/Cruz.pdf [29.03.2016]. 
Cummins, J. (2006). The Imaginative Construction of Self through Multiliteracies Pedagogy. In O. García, T. Skutnabb-Kangas \& M. E. Torres-Guzmán (Hrsg.), Imagining Multilingual Schools. Languages in Education and Globalization (51-68). Clevedon, Buffalo: Multilingual Matters.

Delanoy, W. (2014), Mehrsprachigkeit, Englisch und Literatur(unterricht). Zeitschrift für Interkulturellen Fremdsprachenunterricht 19 (1), 63-76. Verfügbar unter: http://zif.spz.tu-darmstadt.de/ig-191/beitrag/Delanoy.pdf [29.03.2016].

Dörnyei, Z. (2007). Research Methods in Applied Linguistics. Oxford: Oxford University Press.

Elizondo, S. (1977). She. In S. Elizondo (Hrsg.), Libro para batos y chavalas chicanas (62). Berkeley, Calif: Editorial Justa Publications.

Elsner, D. (2012). Multiliteracy Practices als Lernziel: mehrsprachige Gedichte von Pat Mora und Antoine Cassar. In R. Ahrens, M. Eisenmann \& J. Hammer (Hrsg.), Anglophone Literaturdidaktik - Zukunftsperspektiven für den Englischunterricht (409-424). Heidelberg: Winter.

Espaillat, R. P. (1998). Bilingual/Bilingüe. In R. P. Espaillat (Hrsg.), Where horizons go. Poems (1. Aufl.). Kirksville, MO: New Odyssey Press.

Espinosa, A. M. (1953). A Una Niña de Este País. In A. M. Espinosa (Hrsg.), Romancero de Nuevo Méjico (264). Madrid: Consejo Superior de Investigaciones Científicas

Ette, O. (2005). ZwischenWeltenSchreiben. Literaturen obne festen Wobnsitz (ÜberLebenswissen II). Berlin: Kadmos.

Ette, O. (2010). ZusammenLebensWissen. List, Last und Lust literarischer Konvivenz im globalen Maßstab (ÜberLebenswissen III). Berlin: Kadmos.

Flick, U. (2008). Triangulation. Eine Einführung (2. Aufl). Wiesbaden: VS, Verlag für Sozialwissenschaften.

Fuchs, K. A. (2014). Emotionserkennung und Empatbie. Wiesbaden: Springer.

Gafaranga, J. (2007). Talk in two languages. Basingstoke, New York: Palgrave Macmillan.

García, O., Skutnabb-Kangas, T. \& Torres-Guzmán, M. E. (Hrsg.) (2006). Imagining multilingual schools. Languages in education and globalization. Clevedon, England, Buffalo: Multilingual Matters.

Gass, S. M. \& Mackey, A. (2000). Stimulated recall methodology in second language research. Mahwah, NJ: L. Erlbaum Associates.

Gorter, D. \& Cenoz, J. (2011). A Multilingual Approach. Conclusions and Future Perspectives. Afterword. The Modern Language Journal, 95 (3), 442-445.

Grünewald, A. \& Sass, A. (2014). ,Same same but different?‘. Sprachenübergreifende Vernetzung des Englisch- und Spanischunterrichts zur Förderung von Mehrsprachigkeit. Zeitschrift für Interkulturellen Fremdsprachenunterricht, 19 (1), 25-42.

Barbara Hinger (Hg.), Zweite „Tagung der Fachdidaktik“ 2015.

Sprachsensibler Sach-Fach-Unterricht - Sprachen im Sprachunterricht.

(C) 2016 innsbruck university press, ISBN 978-3-903122-51-2, DOI 10.15203/3122-51-2 
Hufeisen, B. \& Neuner, G. (2003). Mehrsprachigkeitskonzept - Tertiärsprachen - Deutsch nach Englisch. Straßburg: Council of Europe Publishing.

Klein, H. G. (2004). Frequently Asked Questions zur romanischen Interkomprehension. In H. G. Klein \& D. Rutke (Hrsg.), Neuere Forschungen zur Europäischen Interkomprehension (15-34). Aachen: Shaker.

Krumm, H.-J., \& Jenkins, E. M. (2001). Kinder und ibre Sprachen - lebendige Mehrsprachigkeit. Sprachenporträts. Wien: Eviva.

López-Gopar, M. E., Núñez-Méndez, O., Sughrua, W. \& Clemente, A. (2013). In pursuit of multilingual practices. Ethnographic accounts of teaching 'English' to Mexican children. International Journal of Multilingualism, 10 (3), 273-291.

Mayr, M. M. (2014). Speaking in Two Voices. Eine empirische Studie zur Rezeption mehrsprachiger Gedichte im Fremdsprachenunterricht. Dissertation, Universität Innsbruck. Innsbruck.

Mayr, M. M. (i.D.). Speaking in Two Voices. Mehrsprachige Gedichte im Fremdsprachenunterricht. In A. Wegner \& I. Dirim (Hrsg.), Mehrsprachigkeit und Bildungsgerechtigkeit. Erkundungen einer didaktischen Perspektive. Leverkusen: Barbara Budrich.

Mayring, P. (2010). Qualitative Inhaltsanalyse. Grundlagen und Techniken (11. Aufl.). Weinheim: Beltz.

Meißner, F.-J. (1995). Umrisse einer Mehrsprachigkeitsdidaktik. In L. Bredella (Hrsg.), Verstehen und Verständigung durch Sprachenlernen? Dokumentation des 15. Kongresses für Fremdsprachendidaktik. (172187). Bochum: Brockmeyer.

Meißner, F.-J. (2008). Mehrsprachigkeitsdidaktik. In R. Tanzmeister (Hrsg.), Lernen - Lebren - Motivation. Fachdidaktik, für Romanisten (63-94). Wien: Praesens.

Meißner, F.-J. \& Reinfried, M. (1998). Mehrsprachigkeit als Aufgabe des Unterrichts romanischer Sprachen. In F.-J. Meißner \& M. Reinfried (Hrsg.), Mehrsprachigkeitsdidaktik. Konzepte, Analysen, Lebrerfabrungen mit romanischen Fremdsprachen (9-22). Tübingen: Narr.

Mertens, J. (2006). Mehrsprachigkeit für alle? In H. Martinez \& M. Reinfried (Hrsg.), Mehrsprachigkeitsdidaktik gestern, beute und morgen. Festschrift für Franz-Joseph Meißner zum 60. Geburtstag (269277). Tübingen: Gunter Narr Verlag.

Reinmann, G. (2015). Qualitative Sozialforschung. Institut für Medien und Bildungstechnologie. Reader. Verfügbar unter: http://qsf.e-learning.imb-uni-augsburg.de/node/503 [29.03.2016].

Schecter, S. R. \& Cummins, J. (Hrsg.) (2003). Multilingual education in practice. Using diversity as a resource. Portsmouth, NH: Heinemann.

Sexl, M. (2003). Literatur und Erfahrung. Ästhetische Erfahrung als Reflexionsinstanz von Alltags- und Berufswissen. Eine empirische Studie. Innsbruck: STUDIA Universitätsverlag. 
van der Walt, C. (2013). Multilingual Higher Education. Beyond English Medium Orientations. Clevedon, England, Buffalo: Multilingual Matters.

Wei, L. (2011). Multilinguality, Multimodality, and Multicompetence. Code- and Modeswitching by Minority Ethnic Children in Complementary Schools. The Modern Language Journal, 95 (iii), 370-384. 


\section{Anhang 1: She (Elizondo 1977)}

She

She,

She speaks English,

She raps English,

She reads English,

She sits English,

Pero quiere en español.

Sueña en español,

piensa en español,

va a la church en español.

Juega en español,

works in English,

siente en español,

drives in English,

hace cariños en español,

runs in English...

se mece en las curvas de sus pasos en español,

Mira, ¡Ay!, Mira en español.

Duerme, duerme chula,

únicamente en español. 


\section{Anhang 2: Bilingual/Bilingüe (Espaillat 1998)}

\section{Bilingual/Bilingüe}

My father liked them separate, one there, one here (allá y aquí), as if aware that words might cut in two his daughter's heart (el corazón) and lock the alien part to what he was - his memory, his name (su nombre) - with a key he could not claim. "English outside this door, Spanish inside," he said, "y basta." But who can divide the world, the word (mundo y palabra) from any child? I knew how to be dumb and stubborn (testaruda): late, in bed, I hoarded secret syllables I read until my tongue (mi lengua) learned to run where his stumbled. And still the heart was one. I like to think he knew that, even when, proud (orgulloso) of his daughter's pen, he stood outside mis versos, half in fear of words he loved but wanted not to hear. 


\section{Anhang 3: A Una Niña de Este País (Espinosa 1953)}

\section{A Una Niña de Este País}

A una niña de este país

yo le hablaba una vez;

yo le hablaba en español,

y ella me hablaba en inglés.

Le dijé: -¿Serás mi amada

y mi corazón también?

Y me dijo la agringada:

-Me no like Mexican men.

Le empecé a hacer cariñitos

en sus dientes de marfil, y me dijo con modito:

-I tell you, you keep still.

Le escribí un papel por nota

le dije:-Entérese de él.

Y me dijo la ingratota:

-I tell you, you go to hell.

-I tell you, te voy a decir,

I'll tell you, yo te diré,

si tú me quieres a mí,

es todo el inglés que sé. 


\section{Anhang 4: Un Beso Is Not a Kiss (Alarcón 1989)}

\section{Un Beso Is Not a Kiss}

Un beso

Es una puerta

Que se abre

Un secreto

Compartido

Un misterio

Con alas

Un beso

No admite

Testigos

Un beso can't

Be captured

Traded

Or sated

Un beso

Is not just

A kiss

Un beso is

More dangerous

Sometimes even fatal 
